Second Draft

\title{
Four Spacetime Dimensions from Multifractal Geometry
}

\author{
Ervin Goldfain
}

Research Scholar, Ronin Institute, Montclair, New Jersey 07043

Email: ervin.goldfain@ronininstitute.org

\begin{abstract}
As paradigm of complex behavior, multifractals describe the underlying geometry of self-similar objects or processes. Building on the connection between entropy and multifractals, we show here that the generalized dimension of geodesic trajectories in General Relativity coincides with the four-dimensionality of classical spacetime.
\end{abstract}

Key words: multifractals, measure theory, Rényi entropy, generalized dimension, geodesic trajectories, relativistic spacetime.

“...most facts about Nature cannot be expressed in terms of the contrast between "black and white", "true and false", or "1 and o". Therefore, those aspects cannot be illustrated by sets; they demand more general mathematical objects that succeed to embody the idea of "shades of gray". Those more general objects are called measures".

From C. J. G. Evertsz and B. B. Mandelbrot, "Multifractal Measures” in Chaos and Fractals, New Frontiers of Science, Springer-Verlag 1992. 


\section{Introduction}

A self-evident proposition of experimental physics is that observations are based on measurements. Any measurable quantity can be expressed in units of mass $(M)$, length $(L)$ and time $(T)$ according to

$$
[x]=M^{\alpha} L^{\beta} T^{\gamma}
$$

In natural units $(\hbar=c=1)$, time and length units coincide, while mass is reciprocal of length or time. Accordingly, (1) turns into

$$
[x]=L^{\delta}
$$

in which

$$
\delta=\beta+\gamma-\alpha
$$

The exponent (3) characterizes the length dimension of quantity $x$ and relates to the fractal or the Hausdorff dimension $\left(D_{H}\right)$. Let the length extent $L$ of $x$ be covered with a mesh of identical boxes of size $l<<L$. The number of boxes required to cover $L$ scales with the ratio $\varepsilon=l / L$ as in

$$
N(\varepsilon) \propto \varepsilon^{-D_{H}}
$$

The Hausdorff dimension satisfies the inequality

$$
d_{T} \leq D_{H} \leq d
$$


where $d_{T}$ stands for the topological dimension (zero for a point, 1 for a continuous and smooth curve, 2 for a continuous smooth surface and so on), whereas $d$ is the dimension of the embedding space. The condition $D_{H} \leq d$ means, for example, that no curve, no matter how convoluted, can do more than fill the space in which is embedded. The lower limit of (5) states the obvious fact that any object must at least have a dimension equal to that of its elements.

The most straightforward example of a one-dimensional object having $D_{H} \in[0,1]$ is the Cantor set. The Sierpinski carpet is a generalization of the Cantor set in two dimensions and its Hausdorff dimension is $D_{H} \in[1,2][2-4]$. The fundamental property of these fractals is self-similarity, or "shape invariance" under rescaling of $\varepsilon$ as in

$$
\varepsilon \rightarrow \varepsilon / r, r \in \mathbf{R}
$$

Self-similarity is closely related to the concept of covariance in field theory, the behavior of the Renormalization Group flow as well as to the geometry of strange attractors defining the onset of turbulence in fluid dynamics [5-7]. It can be also shown that selfsimilarity underlies the physics of self-organized criticality (SOC), a universal manifestation of complex dynamics in large systems outside equilibrium [10].

Unlike simple fractals like the Cantor set and Sierpinski carpet, there are objects where scaling (4) requires two or more dimensions and are referred to as multifractals. These are considered mixtures of fractal objects, each characterized by its Hausdorff dimension. Self-similarity of multifractals is accordingly defined in terms of a multifractal spectrum describing the overall distribution of its fractal dimensions. Next section elaborates on these concepts and similar other tools of multifractal analysis. 


\section{Brief overview of multifractal analysis}

In the general context of complex dynamics, multifractal analysis is considered a theory of self-similar measures [1]. A measure is defined as function that assigns a number to certain subsets of a given set: the number is said to represent the measure of the set. The basic properties of measures are extensions of the familiar geometrical concepts of length, area, and volume, so that - for example - the measure of the union of two disjoint sets is the sum of the measures of the two sets, and the measure of the empty set is zero. Roughly speaking, a self-similar measure is a measure whose geometrical attributes stay unchanged upon arbitrary scaling operations.

To characterize a multifractal whose support has linear extent $L$, let the set be covered with a mesh of identical rectangular boxes of size $l$. Let $P_{i}$ represent the probability measure defined inside the $i^{\text {th }}$ box so that $P_{i}$ vanishes for empty boxes. By analogy with equilibrium statistical mechanics, multifractal analysis is based on a partition function defined as [1-2]

$$
Z_{q}(l, L)=\sum_{i=1}^{N} P_{i}^{q}
$$

The partition function (7) consists of the sum over all boxes of the $q^{\text {th }}$ order of box probabilities, where $-\infty<q<\infty$. One can further simplify (7) by introducing the dimensionless number $\varepsilon=l / L$ so that

$$
Z_{q}(l, L)=Z_{q}(\varepsilon)
$$

Note that for $q=1$, the partition function satisfies the normalization condition 


$$
Z_{1}(\varepsilon)=1
$$

The number of boxes needed to cover the set can be alternatively defined through the power-law scaling

$$
N(\varepsilon) \sim \varepsilon^{-D_{H}}
$$

As indicated in the previous section, $D_{H}$ denotes the Hausdorff dimension of the set, which is adequate for characterization of mono-fractals. In general, the quantitative description of multifractal measures requires replacing $D_{H}$ with a continuous LipschitzHölder (LH) exponent $\alpha$ according to

$$
P_{i} \sim \varepsilon^{\alpha}, \quad 0<\alpha \in\left[\alpha_{\min }, \alpha_{\max }\right]<\infty
$$

By analogy with (10), the number of boxes of size $\varepsilon$ having the LH exponent $\alpha$ is given by

$$
N_{\varepsilon}(\alpha) \sim \varepsilon^{-f(\alpha)}
$$

where the distribution of LH exponents follows the multifractal spectrum $f(\alpha)$. The meaning of (12) is that there are infinitely many subsets of boxes having the LH exponent $\alpha$ in the limit $\varepsilon \rightarrow 0$.

Denoting the number of boxes for which $\alpha<\alpha_{i}<\alpha+d \alpha$ as $N_{\varepsilon}(\alpha) d \alpha$, the contribution of the subset of boxes with $\alpha_{i} \in[\alpha, \alpha+d \alpha]$ to the partition function is $N_{\varepsilon}(\alpha)\left(\varepsilon^{\alpha}\right)^{q} d \alpha$ and thus 


$$
Z_{q}(\varepsilon)=\int N_{\varepsilon}(\alpha)\left(\varepsilon^{\alpha}\right)^{q} d \alpha
$$

By (12) and (13), we obtain

$$
Z_{q}(\varepsilon)=\int \varepsilon^{q \alpha-f(\alpha)} d \alpha
$$

Assume now that each box of dimension $\varepsilon$ is rescaled by a box-dependent factor $r_{j}$. Let $p_{j}$ denote the probability associated with the $j^{\text {th }}$ rescaled version of the original box whose dimension is

$$
\varepsilon_{j}=\frac{\varepsilon}{r_{j}}
$$

so that

$$
\sum_{j=1}^{N} p_{j}=1
$$

The rescaling process can be recursively continued an arbitrary number of times. Each rescaled version of the original partition carries an amount $p_{j}$ of the total measure. The similarity property of the rescaling process leads to the condition [2]

$$
Z_{q, j}(\varepsilon)=p_{j}^{q} Z_{q}\left(\frac{\varepsilon}{r_{j}}\right)
$$

where $Z_{q, j}(\varepsilon)$ is the rescaled value of the partition function (14). Additivity of (14) implies that 


$$
Z_{q}(\varepsilon)=\sum_{j=1}^{N} Z_{q, j}(\varepsilon)
$$

Considering the above relationships, one arrives at the condition [2]

$$
\sum_{j=1}^{N} p_{j}^{q} r_{j}^{\tau(q)}=1
$$

in which

$$
\tau(q)=f(\alpha)-q \alpha
$$

An important result of multifractal analysis is that, for $\varepsilon \rightarrow 0$, the partition function follows the power law [2]

$$
Z_{q}(\varepsilon) \propto \varepsilon^{(q-1) D_{q}}
$$

where $D_{q}$ represents the order $q$ generalized dimension. The factor $(q-1)$ is explicitly pulled out of the exponent in (21) to enforce the normalization condition $Z_{1}(\varepsilon)=1$. The topic of generalized dimensions is elaborated upon in the Appendix section.

\section{Geodesic trajectories and multifractal geometry}

Geodesic trajectories in General Relativity arise from the interval equation [8]

$$
\sum_{\mu=0}^{3} \sum_{v=0}^{3} g_{\mu v} \frac{d x^{\mu}}{d s} \frac{d x^{v}}{d s}=1
$$

subject to the constraint 


$$
\sum_{v=0}^{3} g^{\mu v} g_{v \rho}=\delta_{\rho}^{\mu}=\left\{\begin{array}{l}
1, \mu=\rho \\
0, \mu \neq \rho
\end{array}\right.
$$

Consider the limit of low four-velocities

$$
\frac{d x^{\mu}}{d s}<1, \quad \mu=0,1,2,3
$$

and compare (27) - (29) to (19). One is led to the following mapping

$$
p_{j} \Rightarrow g^{\mu v} g_{v \rho}, \quad g^{\mu v} \Rightarrow p_{j}^{1 / 2} \Rightarrow q=1 / 2, \quad r_{j} \Rightarrow \frac{d x^{\mu}}{d s}, \tau(q)=2
$$

In this context, metric coefficients $g^{\mu \nu}$ are interpreted as analogs of "probability amplitudes" and the components of the four-velocity as analogs of scaling ratios $r_{j}$. Replacing (30) in (19) and accounting for (A5) recovers the four-dimensionality of classical spacetime in the form

$$
D_{1 / 2}=4
$$

\section{Concluding remarks}

It is well known that the union of space and time forms the basis of Special Relativity and is carried over to General Relativity. The four-dimensionality of relativistic spacetime in the classical theory of gravitation is therefore assumed from the outset.

In contrast, the approach taken here derives the existence of four spacetime dimensions from the fundamental connection between continuous dimensionality and entropy in multifractal geometry. 
Applying this connection to the expression of the line element in General Relativity leads to a derivation of the number of spacetime dimensions from the entropy. The key observation here is that the derivation is only possible in the framework of multifractal geometry, where dimensions represent a manifestation of entropy.

In closing, we bring up several observations:

a) Combined use of (31) and (A3) indicates that the limit $\varepsilon \rightarrow 0$ matches the transition $d \rightarrow D_{1 / 2}$. It follows that the dimensional deviation $\varepsilon=4-d<<1$ may be interpreted as a scale-dependent and continuous parameter defining the concept of minimal fractal manifold [6].

b) (A4) shows that the Rényi entropy asymptotically diverges in the classical spacetime limit $\varepsilon \rightarrow 0$. This finding lends support to the idea that (A4) reflects, in fact, the second law of Thermodynamics.

c) There is a fundamental distinction between Rényi entropy and the Beckenstein entropy, the latter referring to the event horizon of Black Holes. While Rényi entropy relates to the number of fuzzy bits ("shades of grey") in a given spacetime region, Beckenstein entropy relates to the number of binary bits ( 1 or 0 ) holographically encoded on the horizon surface [11]. Digging deeper into this difference in concepts is beyond the scope of the paper.

\section{APPENDIX A}

Generalized dimensions are associated with the concept of Rényi entropy which is defined as $[2,9]$ 


$$
S_{q}=\frac{1}{q-1} \log \sum_{j=1}^{N} p_{j}^{q}
$$

(A1) recovers the standard thermodynamic entropy of a discrete probability distribution in the limit $q \rightarrow 1$, i.e.

$$
S_{1}=-\sum_{j=1}^{N} p_{j} \log p_{j}
$$

The relationship between the generalized dimensions $D_{q}$ and Rényi entropy is given by

$$
D_{q}=-\lim _{\varepsilon \rightarrow 0} \frac{S_{q}}{\log \varepsilon}
$$

or

$$
\lim _{\varepsilon \rightarrow 0}\left(\varepsilon^{-D_{q}}\right) \propto \exp \left(S_{q}\right)
$$

For $q=0$, one obtains $D_{0}=D_{H}$ and the generalized entropy coincides with the Hausdorff dimension. The information dimension $D_{1}$ corresponds to $q=1$ and the associated entropy $S_{1}$ describes the loss of information in the time evolution of chaotic systems. Likewise, the correlation dimension $D_{2}$ encodes the probability of finding two members of the multifractal set within a distance $O(\varepsilon)$. In general, the relevant dimensions of a multifractal are ordered as in $D_{H}>D_{1}>D_{2}$. Comparative inspection of (14) and (21) reveals the following correspondence [2]

$$
\tau(q)=(1-q) D_{q}=f(\alpha)-q \alpha
$$


(A5) acts as a Legendre transform between parameters $q, D_{q}$ and $\alpha, f(\alpha)$. This transformation enables a one-to-one mapping between multifractal geometry and Lagrangian field theory [10].

\section{$\underline{\text { References }}$}

1. Evertsz, C. J. G and Mandelbrot, B. B., "Multifractal Measures", in Chaos and Fractals, New Frontiers of Science, Springer-Verlag 1992.

2. https://www.researchgate.net/publication/272400210

3. https://arxiv.org/pdf/1606.02957.pdf

4. Peitgen, H. O. et al, Chaos and Fractals, New Frontiers of Science, Springer-Verlag 1992.

5. https://www.researchgate.net/publication/342876637

6. https://www.researchgate.net/publication/278849474

7. https://www.researchgate.net/publication/344227402

8. see e.g., Dirac, P. A. M., “General Theory of Relativity”, Princeton Univ. Press, 1996.

9. https://www.researchgate.net/publication/336047781

10. https://www.researchgate.net/publication/343425482

11. http://www.scholarpedia.org/article/Bekenstein-Hawking entropy 